\title{
Investigation of Thermal Flow Sensor Based on Laser Induced Fluorescence Technique
}

\author{
Bin $\mathrm{Xu}$, Nam-Trung Nguyen, Teck Neng Wong* \\ School of Mechanical and Aerospace, Nanyang Technological University \\ 50 Nanyang, Avenue, Singapore 639798
}

\begin{abstract}
In this paper, the heat transfer characteristics of a thermal flow sensor are investigated experimentally and numerically. DI-water is employed as the working fluid. Operation mode with a constant heater temperature is considered in our experiment. The main part of the thermal flow sensor is a cylindrical copper heater. Laser induced fluorescence (LIF) method is used to measure the full temperature field of the fluid in the microchannel. Different flowrates were studied to investigate the temperature distribution in the microchannel. The flow direction and velocity can be predicted based on the temperature distribution. A numerical simulation of conjugate forced convection-conduction heat transfer is employed to investigate the heat transfer processes in the thermal flow sensor. The measured temperature profiles along the central axis of the microchannel and the temperature differences between two positions upstream and downstream at different flowrates were compared with numerical simulation results. The results show that the LIF method is suitable for temperature characterization in microscale in general and for the characterization of thermal flow sensor in particular.
\end{abstract}

Key words: thermal flow sensor, flowrate, Laser Induced Fluorescence

*Corresponding author. E-mail: mtnwong@,ntu.edu.sg 


\section{Introduction}

Flow measurement is of great importance in many applications of microfluidics such as pharmaceutical industry, medical and (bio)chemical analysis. Many kinds of micro flow sensors based on different measuring principles have been proposed [1-5]. Conventional micro flow sensor concepts such as pyroelectric elements, pn-junctions and resonating microbridges need complicated fabrication processes and extensive measurement setups [6].

Thermal flow sensor usually employs a temperature field to measure the velocity and direction of a fluid in a microfluidic system. This flow sensing concept is popular due to its low cost and high performance characteristics [7]. Hot wires and hot films are the two simplest forms of a thermal flow sensor, which can only measure a local point velocity. The more sensitive calorimetric concept uses a miniaturized heat source and two temperature sensors located symmetrically on both side of the heat source [8]. The flow velocity can be calibrated by measuring the temperature difference $(\Delta T)$ between two positions upstream and downstream of the heat source. Its working principle is to measure the heat transfer from the heat source to the surrounding fluid, which is carried away by convection [9]. The rate of heat transfer is proportional to the mass flow rate in the microchannel. A schematic illustration of the working principle of a thermal flow sensor is shown in Figure 1, where two temperature sensing element are put at both sides of a central heater (upstream and down stream) [10].

Many research workers in the past focus on of the calorimetric thermal flow 
sensor due to its high sensitivity. Sabate et al. [11] studied three different sensing materials for integrated thermal flow sensors, including platinum, nickel and polysilicon. The sensitivity, electrical stability and fabrication technology compatibility of these sensing materials were investigated. Rodrigues et al. [12] proposed a new thermal flow sensor for gas and liquid flow measurements which was based on calorimetric and time of flight principles with six free standing microfilaments. Furjes et al. [10] employed numerical simulation to obtain an optimum geometric design, including device dimensions and channel depth. Nguyen [7] presented an unified two-dimensional forced convection theoretical model with a laminar flow for different thermal flow sensor concepts. A novel thermal flow sensor with a single heater and an array of temperature sensors was designed and tested to validate the analytical model. Shin et al. [13] conducted experiments and numerical simulation to investigate various design options for the thermal flow sensor based on the concept of calorimetric sensing. Kaltsas et al. [14] presented the development and evaluation of a smart flow measurement system based on a thermal flow sensor which also employed the calorimetric principle. Beigelbeck et al. [15] made use of a micromachined calorimetric sensor to measure the thermal conductivity and diffusivity of the fluids, regardless of their motion state. Shuo et al. [16] investigated the heat transfer process of the micro thermal flow sensor numerically. The heat conduction in the substrate and different positions of temperature sensors were found to have great influence on the sensor's performance.

The accuracy of the calorimetric concept is limited by the drift in the electrical 
resistance of the thin film layers and the influence of external temperature gradients across the sensor element [17]. In order to overcome the limit of this kind of thermal flow sensor, some researchers make an attempt to solve these limits. Difkstra et al. [17] designed a highly sensitive micro thermal flow sensor using thermopiles combined with a controlled heater resistor eliminated the drift in the electrical resistance. The special layout of the microchannel compensated the influence of external temperature gradient on the temperature sensor. Wiegerink et al. [18] developed a micro thermal and Coriolis flow sensor based on the measurement of Coriolis forces, which were independent of temperature and fluid properties.

This paper presents a micro thermal flow sensor for liquid flows. The flow sensor consists of a heating element embedded in a straight microchannel with rectangular cross section. Laser Induced Fluorescence (LIF) method is employed to measure the full temperature field in the microchannel. Compared to conventional temperature measurement based on sensing elements located at fixed points in the field, LIF method can directly provide information of the temperature distribution for the whole field within the microchannel. Experiments are carried out to show the feasibility of using LIF method for a micro thermal flow sensor. A two-dimensional numerical simulation is employed to provide a better understanding of the conjugate forced convection-conduction heat transfer processes of a micro thermal flow sensor.

\section{Experiment}

\subsection{Fluorescence imaging setup}


The fluorescence imaging setup consists of four main components: an illumination system, an optical system, a coupled charge device (CCD) camera and a personal computer (PC) based control system. A mercury lamp with the wave length of $540 \mathrm{~nm}$ was employed as the illumination source for the fluorescence imaging setup.

The Nikon inverted microscope (Model ECLIPSE TE2000-S) with a set of epi-fluorescent attachments was used as the optical system. Excitation filter, dichroic mirror and emission filter constitute the filter cube. Emission filters were used in the measurements to select the specific emission wavelength of the sample and to remove traces of excitation light.

An interline transfer CCD camera (Sony ICX 084) was used for recording the images. The resolution of the camera is $1324 \times 1024$ pixels with 16 bits grayscale. The active area of the CCD sensor is $4 \mathrm{~mm} \times 3 \mathrm{~mm}$. The exposure time for recording the image is $70 \mathrm{~ms}$.

\subsection{Calibration of temperature measurement}

The schematic of the experimental setup for the calibration of temperature measurement is shown in Figure 2. The calibration was used to determine the relationship between the fluorescence intensity and temperature. The microchannel has a cross section of $1000 \mu \mathrm{m} \times 500 \mu \mathrm{m}$. Rhodamine B solution $(0.025 \mathrm{~g} / 100 \mathrm{ml})$ was introduced into the inlet port A by a single syringe pump (Cole-Parmer, 74900-05, $0.2 \mu \mathrm{l} / \mathrm{h}$ to $500 \mathrm{l} / \mathrm{h}$, accuracy of $0.5 \%$ ). A power supply (GW Model GPC- 30300) was used to supply voltage to the thermoelectric module (TEC) to heat up the 
Rhodamine B solution in the microchannel. The voltage was supplied to enable both heating and cooling in the microchannel.

The thermoelectric module was placed on the square slot on the top PMMA plate to heat the Rhodamine B solution by varying the voltages. The temperature of the solution increases with increasing voltage. The images of the Rhodamine B solution in the microchannel at different temperatures were captured with the camera attached to the microscope. Each captured image was generated by 30 sequential video frames. For the calibration curve, the fluorescence intensity for the image at each temperature was determined by averaging the intensity value (after background subtraction) of all the pixels of the corresponding image. The resulting intensity (normalized by the intensity at $25^{\circ} \mathrm{C}$ ) versus temperature is shown in Figure 3. A calibration curve is generated to accurately describe the dependence of Rhodamine B fluorescence on temperature. The calibration curve is mathematically expressed by

$$
T=A_{0}+A_{1} I+A_{2} I^{2}+A_{3} I^{3}
$$

where $\mathrm{T}$ and I are the temperature and normalized fluorescence intensity, respectively, and $A_{0}=139.84{ }^{\circ} \mathrm{C}, A_{1}=-212.02{ }^{\circ} \mathrm{C}, A_{2}=128.66{ }^{\circ} \mathrm{C}, A_{3}=-31.62{ }^{\circ} \mathrm{C}$.

\subsection{Experiment on thermal flow sensor}

The thermal flow sensor used in the experiment is shown in Figure 4(a), while the schematic illustration of focused area captured by the CCD camera is shown in Figure 4(b). The fabrication is based on the lamination thermal bonding technique [19]. In this method, three polymethylmethacrylate $($ PMMA) plates $(50 \mathrm{~mm} \times 25 \mathrm{~mm})$ were bonded together to form a closed microfluidic channel with inlet and outlet holes. 
Thus, the middle PMMA defines the depth of the channel. A copper cylinder was inserted into the microchannel from the hole of the top PMMA plate. An electric resistance wire was wrapped on the copper cylinder circumference. The straight channel has a cross section of $1000 \mu \mathrm{m} \times 2000 \mu \mathrm{m}$ and a length of $3 \mathrm{~cm}$. DI-water with Rhodamine B solution $(0.025 \mathrm{~g} / 100 \mathrm{ml})$ was introduced into inlet port by a syringe pump. A low voltage power supply is used to supply voltage to the electric resistance wire to enable heating of the copper cylinder. The images of the Rhodamine B solution around the heater for different flow rates were captured by the CCD camera.

\section{Two-dimensional numerical simulation}

The schematic of the computational domain is shown in Figure 4(c). The model consists of two PMMA plates, of which a heater is placed in the middle of the microchannel. The heat transfer process of a micro thermal flow sensor is a conjugate forced convection-conduction problem. Therefore, it is needed to take into consideration of the heat conduction in the PMMA plates and the heat convection in the fluid. Furthermore, the temperature fields obtained from the numerical simulation can be used to confirm the trend of experimental results.

The heat transfer process of a thermal flow sensor occurs in two regions including the solid region (PMMA plates) and the fluid region (fluid flow). The temperature distribution in the solid region is governed by the two-dimensional heat conduction equation while the temperature distribution in the fluid region is governed by the two-dimensional heat conduction-convection equation. In the numerical simulation 
model, laminar, incompressible, Newtonian fluid and steady flow are assumed. The density, specific heat, thermal conductivity and viscosity of DI-water are allowed to vary with temperature [20].

\subsection{Governing equations}

The governing equations for the fluid flow and heat transfer characteristics of a thermal flow senor can be written in a Cartesian coordinate system as follow.

\subsubsection{Fluid velocity field}

With the assumption of the incompressible Newtonian fluid, the fluid velocity field is described by the continuity and Navier-Stokes equations.

$$
\begin{gathered}
\nabla \cdot \vec{V}=0 \\
\rho(T)\left(\frac{\partial \vec{V}}{\partial t}+\vec{V} \cdot \nabla \vec{V}\right)=-\nabla p+\nabla(\mu(T) \nabla \vec{V})
\end{gathered}
$$

Where $\vec{V}$ is the fluid velocity vector, $\rho$ and $p$ are the fluid density and hydrodynamic pressure respectively, $\rho(T)$ and $\mu(T)$ are the fluid density and viscosity respectively, whose unit are $\mathrm{kgm}^{-3}$ and $\mathrm{Pa} \cdot \mathrm{s}$. Their dependence on temperature are given as follows,

$$
\begin{gathered}
\rho=\frac{999.8+18.2 \times T-7.9 \times 10^{-3} \times T^{2}-5.5 \times 10^{-5} \times T^{3}+1.5 \times 10^{-7} \times T^{4}-3.9 \times 10^{-10} T^{5}}{1+1.8 \times 10^{-2} \times T} \\
\mu=2.414 \times 10^{-5} \times 10^{247.8 /(T-140)}
\end{gathered}
$$

\subsubsection{Fluid and channel wall temperature field}


The temperature field in the microchannel is governed by the energy equation expressed as

$$
\rho(T) c_{p}(T)\left(\frac{\partial T}{\partial t}+\vec{V} \cdot \nabla T\right)=\nabla(k(T) \nabla T)
$$

where $c_{p}$ and $k$ are specific heat and the thermal conductivity of the working fluid respectively, whose unit are $\mathrm{Jkg}^{-1} \mathrm{~K}^{-1}$ and $\mathrm{Wm}^{-1} \mathrm{~K}^{-1}$. Their dependence on temperature are given as follows,

$$
\begin{gathered}
c_{p}=8958.9-40.535 \times T+0.11243 \times T^{2}-1.0138 \times 10^{-4} \times T^{3} \\
k=-0.58166+6.3555 \times 10^{-3} \times T-7.9643 \times 10^{-6} \times T^{2}
\end{gathered}
$$

In addition, since the thermal boundary condition on the channel wall is unknown, a conjugate heat transfer problem has to be solved by simultaneously considering the heat conduction in the channel wall. The temperature field in the channel wall is governed by the heat conduction equation expressed as

$$
\rho_{s} c_{p s} \frac{\partial T}{\partial t}=k_{s} \nabla^{2} T
$$

where $\rho_{s}, c_{p s}, k_{s}$ are the specific heat, density, and thermal conductivity of the microchannel wall (PMMA plate), respectively.

\subsection{Initial and boundary conditions}

The initial and boundary conditions for the fluid flow and heat transfer in the microchannel are:

At the inlet $(x=-L,-H<y<H), T_{i n}=25^{\circ} \mathrm{C}, u=u_{i n}, \quad v=0$

At the outlet $(x=L,-H<y<H), \frac{\partial T}{\partial x}=0, \frac{\partial u}{\partial x}=0, \frac{\partial v}{\partial x}=0$

In the fluid region, $T_{i}=25^{\circ} \mathrm{C}, \quad u_{i}=0, v_{i}=0$ 
The initial and boundary conditions for the heat conduction in the solid region are:

At the left wall $(x=-L,-W<y<-H, H<y<W), T=25^{\circ} \mathrm{C}$

At the right wall $(x=L,-W<y<-H, H<y<W), T=25^{\circ} \mathrm{C}$

At the top and bottom wall $(y=W, y=-W), \frac{\partial T}{\partial y}=0$

In the solid region, $T_{i}=25^{\circ} \mathrm{C}$

The boundary conditions at the interface between the solid and the fluid regions are governed by the continuity of heat flux and temperature:

At the upper interface $(y=H), T_{s}=T_{f}, k_{s} \frac{\partial T_{s}}{\partial y}=k_{f} \frac{\partial T_{f}}{\partial y}$

At the lower interface $(y=-H), T_{s}=T_{f}, k_{s} \frac{\partial T_{s}}{\partial y}=k_{f} \frac{\partial T_{f}}{\partial y}$

At all the channel walls, the no-slip boundary condition is applied to the momentum equation. A constant heating temperature is applied at the circumference of the heater.

\subsection{Numerical method}

Since the governing equations for the velocity and temperature fields are strongly coupled in the temperature dependent parameters, the numerical method is inherently used to solve this coupled problem. COMSOL Multiphysics ${ }^{\mathrm{TM}}$ was employed to numerically solve governing equations (2) - (9) to obtain the fluid velocity field, and the temperature field.

A grid independence test was conducted by using different numbers of 
unstructured grid. Three different grid numbers as 1284, 5136 and 20544 are employed with a set of parameters as $\mathrm{Q}=0 \mu \mathrm{l} / \mathrm{h}, \quad k_{s}=0.25 \mathrm{Wm}^{-1} \mathrm{~K}^{-1}$, $k_{f}=0.6 \mathrm{Wm}^{-1} \mathrm{~K}^{-1}, T_{h}=50{ }^{\circ} \mathrm{C}$. The differences in $u, v$ and $T$ at all grid points are less than $1 \%$, which means the numerical solution is grid independent. Therefore, the numerical method is considered to be appropriate for the simulation of our conjugate convection-conduction heat transfer problem.

\section{Results and discussion}

Figure 5 shows the temperature distribution at different flowrates. The experimental results are on the left column, while the numerical simulation results are on the right column. At zero flowrate $(\mathrm{Q}=0 \mu \mathrm{l} / \mathrm{h})$, a symmetrical temperature distribution is obtained, which corresponds to the special case of pure heat conduction (Figure 5(a)). At a certain flowrate in the presence of an incoming flow, the symmetrical temperature distribution around the heater will change to an asymmetric temperature distribution. Figures 5 (b) and 5(c) show the variation of temperature contours around the heater corresponding to different flowrates. As the flowrate increases, the temperature contour lines of upstream will be lowered and narrowed, which indicates that the temperature gradient of the heater upstream becomes steeper. As the flowrate increases, the heat generated by the heater will be carried away downstream by convection. The amount of the transferred heat is proportional to the flowrate. At higher flowrate, the incoming flow will have less residence time to be heated by the heater. Conversely, increasing the flowrate will broaden the temperature 
contour lines of the heater downstream, which indicates that the temperature field downstream becomes more gradual. The higher flowrate, the more heat is carried downstream. The numerical simulation results (Figure 5(d)-(f)) show the same trend as the experimental results.

To have a better understanding of the thermal characteristics of a thermal flow sensor, temperature profiles along the center line of the microchannel with different flowrates are plotted in Figure 6. Figure 6(a) shows the temperature profile along the center line of the microchannel with flowrate equal to zero. In this figure, the slope of temperature profile in the upstream region was the same as in the downstream region. Such thermal symmetry behavior is caused by the central position of the heater in the microchannel. Combined with symmetrical boundary conditions, the temperature distribution in the microchannel is inherently symmetric. In the presence of a coming flow in the microchannel, the thermal symmetry behavior is disturbed. Figure $6(\mathrm{~b})$ shows the temperature profile along the center line of the microchannel with flowrate equal to $1000 \mu \mathrm{l} / \mathrm{h}$. In the presence of a coming flow, some heat was transferred to the upstream region will be carried to the downstream region. As a result, the upstream temperature decreases while the downstream temperature increases. The cooling effect appears in the upstream region. Temperature difference between upstream and downstream occurs. The slope of temperature profile in the heater upstream region becomes steeper than the heater downstream region. Figure 6(c) shows the temperature profile along the center line of the microchannel with flowrate equals to $2000 \mu \mathrm{l} / \mathrm{h}$. As the flow rate becomes larger, more cold fluid can pass through the 
heater without being heated. The cooling effect of the upstream becomes more obvious. Thus the slope of the upstream temperature profile will becomes much steeper than the slope of the downstream temperature profile.

The corresponding temperature difference caused by increasing the flowrate between two positions upstream and downstream is shown in Figure 7. Four different distance differences between two positions upstream and downstream are selected to show the thermal characteristics of a thermal flow sensor: $2 d, 3 d, 4 d$ and $5 d$. Figure 7(a) shows the temperature difference between upstream and downstream positions (1-1section). It is shown that the temperature difference becomes larger as the flow rate increases. As discussed above, increasing the flowrate disturbs the thermal symmetry characteristic. Asymmetric slope of temperature profiles in the heater upstream and downstream occurs. The larger the flowrate, the larger difference of the slope will occur. Thus, the temperature difference will increases as the flowrate increases. Figure 7(b)-(d) show the temperature difference between upstream and downstream positions (2-2, 3-3 and 4-4 sections). These results show the same trend as the Figure 7(a). The difference is that the slope of temperature difference increases as increase of the distance between two positions upstream and downstream. This confirms that the cooling effect upstream is obvious. The cooling effect increases with distance away from the heater.

\section{Conclusions}

The heat transfer characteristics of a thermal flow sensor were investigated 
experimentally and numerically. The important results obtained are:

(1) The entire heat transfer process of a thermal flow sensor is a conjugate forced convection-conduction heat transfer problem. The heat conduction in the surrounding channel wall should be taken into consideration.

(2) The flowrate plays an important role in the heat transfer process of a thermal flow sensor. Different flowrates correspond to different temperature field.

(3) LIF method can be used to measure the full temperature field in the microchannel. Based on the temperature field, we can judge the velocity direction of working fluid in the microchannel. 


\section{References}

[1]. Belal, M., et al.: An interferometric current sensor based on optical fiber micro wires. Optics Express, 2010, 18, 19951-19956.

[2]. Ito, Y., T. Higuchi, and K. Takahashi: Submicroscale flow sensor employing suspended hot film with carbon nanotube fins. Journal of Thermal Science and Technology, 2010, 5, 51-60.

[3]. Moirangthem, R.S., et al.: Surface plasmon resonance ellipsometry based sensor for studying biomolecular interaction. Biosensors and Bioelectronics, 2010, 25, 2633-2638.

[4]. Seo, Y.H. and B.H. Kim: A self-resonant micro flow velocity sensor based on a resonant frequency shift by flow-induced vibration. Journal of Micromechanics and Microengineering, 2010, 20.

[5]. Lee, C.Y., et al.: Use of flexible micro-temperature sensor to determine temperature in situ and to simulate a proton exchange membrane fuel cell. Journal of Power Sources, 2011, 196, 228-234.

[6]. Kohl, F., et al.: Development of miniaturized semiconductor flow sensors. Measurement: Journal of the International Measurement Confederation, 2003, 33, 109-119.

[7]. Nguyen, N.T.: A novel thermal sensor concept for flow direction and flow velocity. IEEE Sensors Journal, 2005, 5, 1224-1234.

[8]. Talic, A., et al.: Novel Thermal Flow Sensors Based on a Wheatstone Bridge Read-out. Procedia Chemistry, 2009, 1, 136-139.

[9]. Elwenspoek, M.: Thermal flow micro sensors. Proceedings of the International Semiconductor Conference, CAS, 1999, 2, 423-435.

[10]. Furjes, P., et al.: Thermal characterisation of a direction dependent flow sensor. Sensors and Actuators, A: Physical, 2004, 115, 417-423.

[11]. Sabate, N., et al. A test structure for the design of thermal flow sensors. in IEEE International Conference on Microelectronic Test Structures. 2002.

[12]. Rodrigues, R.J. and R. Furlan: Design of microsensor for gases and liquids flow measurements. Microelectronics Journal, 2003, 34, 709-711.

[13]. Shin, W.C. and R.S. Besser: A micromachined thin-film gas flow sensor for microchemical reactors. Journal of Micromechanics and Microengineering, 2006, 16, 731-741.

[14]. Kaltsas, G., et al.: A smart flow measurement system for flow evaluation with multiple signals in different operation modes. Measurement Science and Technology, 2007, 18, 3617-3624.

[15]. Beigelbeck, R., et al. Thermal property determination of laminar-flowing fluids utilizing the frequency response of a calorimetric flow sensor. in Proceedings of IEEE Sensors. 2008.

[16]. Shuo, H., et al. A numerical investigation of conjugate forced heat transfer for a micro thermal flow sensor. in IEEE/ASME International Conference on Advanced Intelligent Mechatronics, AIM. 2009.

[17]. Difkstra, M., et al. Ambient temperature-Gradient compensated low-Drief thermopile flow sensor. in Proceedings of the IEEE International Conference on Micro Electro Mechanical Systems (MEMS). 2009.

[18]. Wiegerink, R.J., et al.: Thermal and Coriolis type micro flow sensors based on surface channel technology. Procedia Chemistry, 2009, 1, 1455-1458.

[19]. Sun, Y., Y.C. Kwok, and N.T. Nguyen: Low-pressure, high-temperature thermal bonding of polymeric microfluidic devices and their applications for electrophoretic separation. Journal 
of Micromechanics and Microengineering, 2006, 16, 1681-1688.

[20]. Incropera, F.P., Liquid Cooling of Electronic Devices by Single-Phase Convection. John Wiley \& Sons, Inc: New York, 1999 


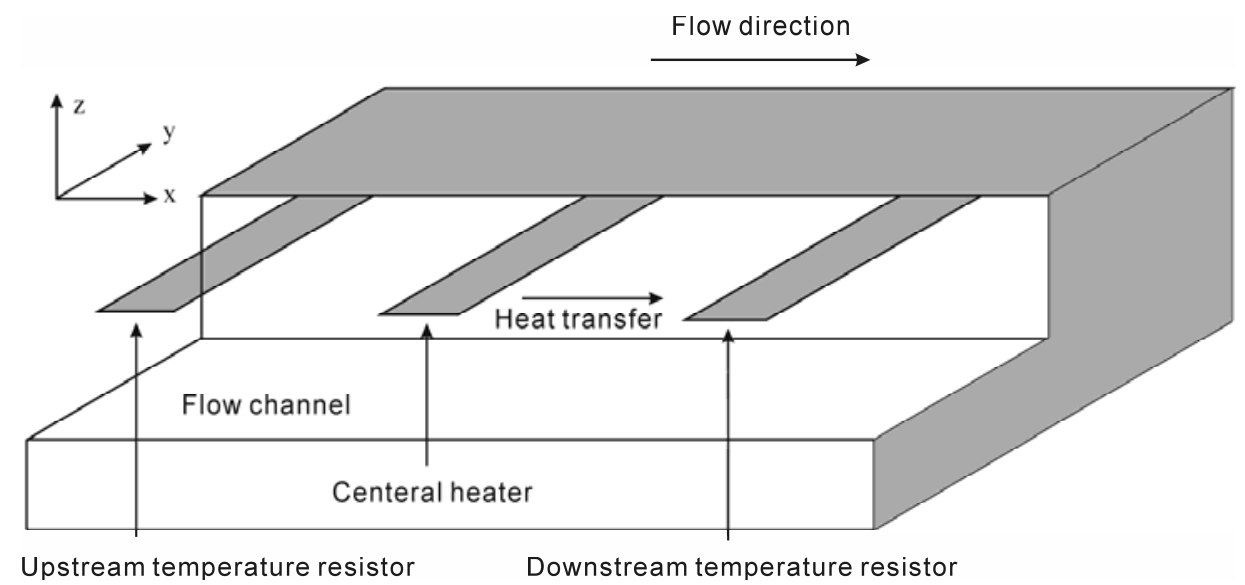

Figure 1. Schematic illustration of the working principle of a thermal flow sensor [10]

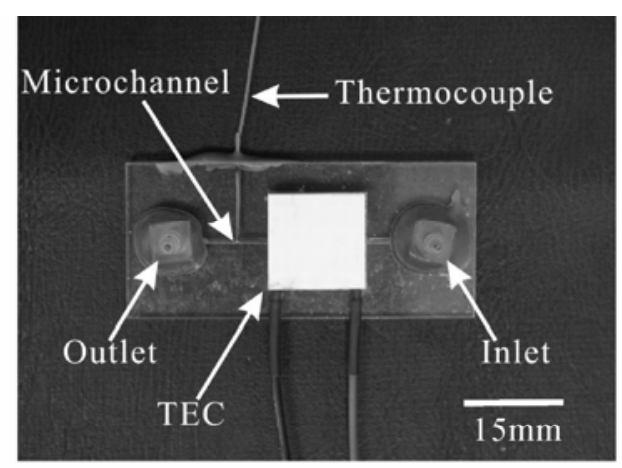

(a)

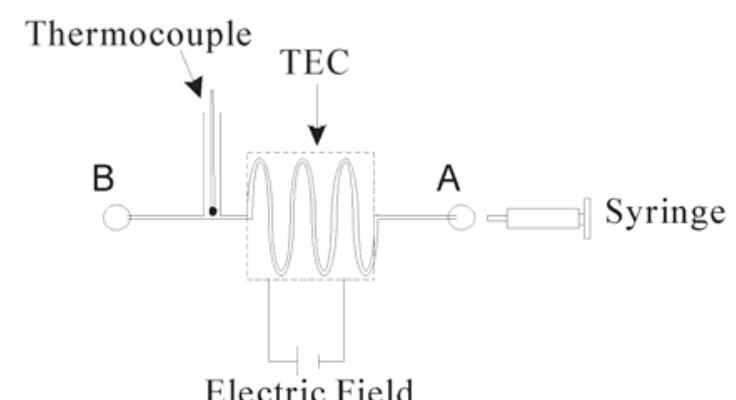

(b)

Figure 2. Schematic illustration of experimental setup for temperature calibration. (a) Fabricated device in the calibration, (b) Schematic representation of the calibration of temperature measurement 


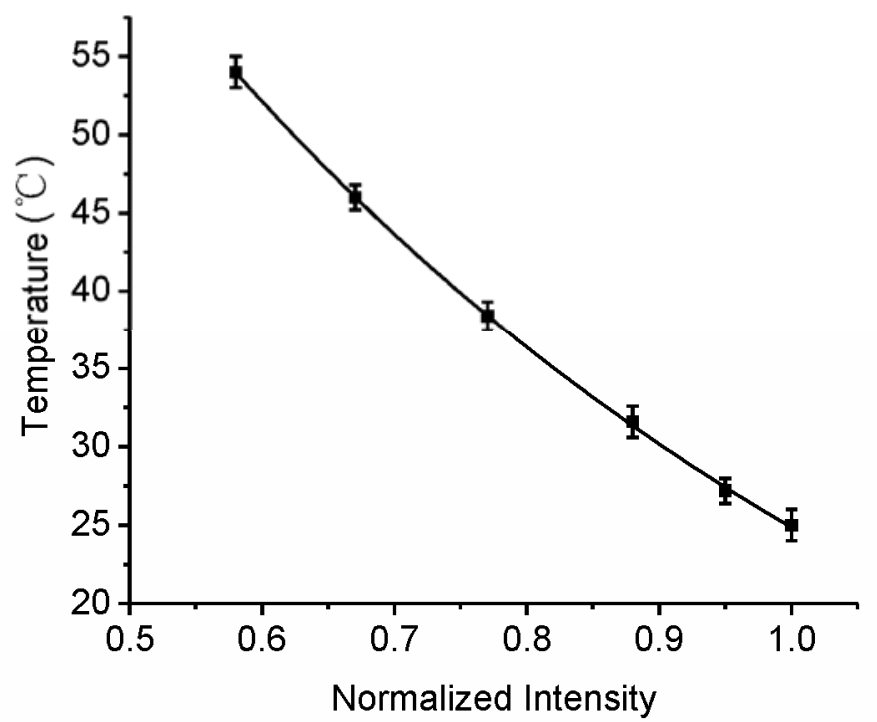

Figure 3. Normalized fluorescence intensity as a function of temperature 


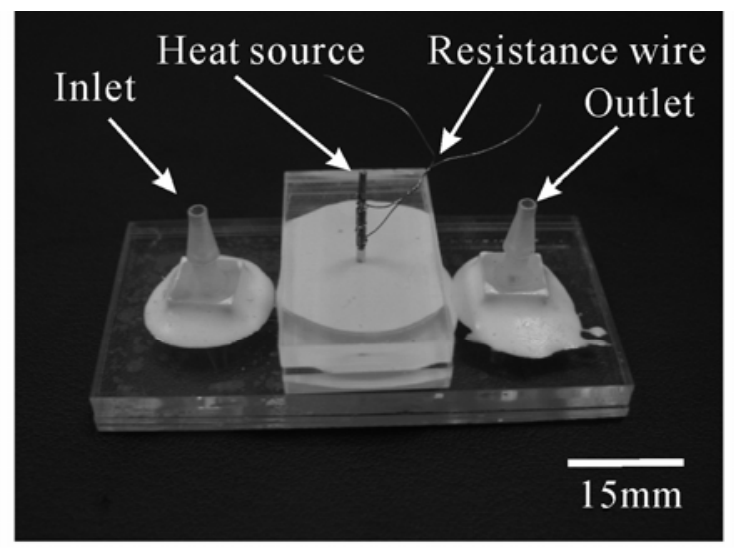

(a)

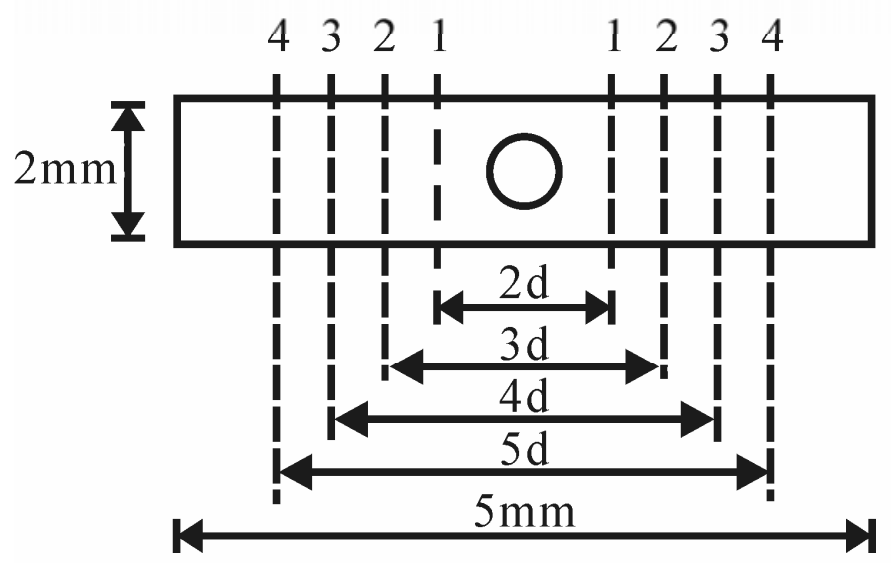

(b)

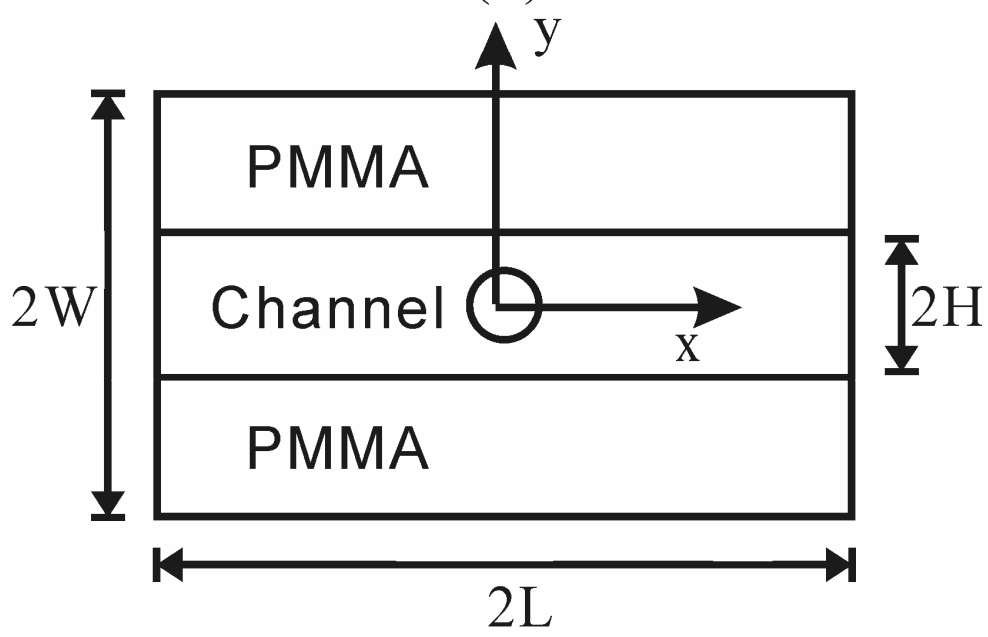

(c)

Figure 4. Schematic illustration of experimental setup of thermal flow sensor. (a) The fabricated device used in the experiment, (b) Schematic illustration of focused area captured by CCD camera, (c) Computational domain (including two PMMA plates, microchannel and a heating element) 

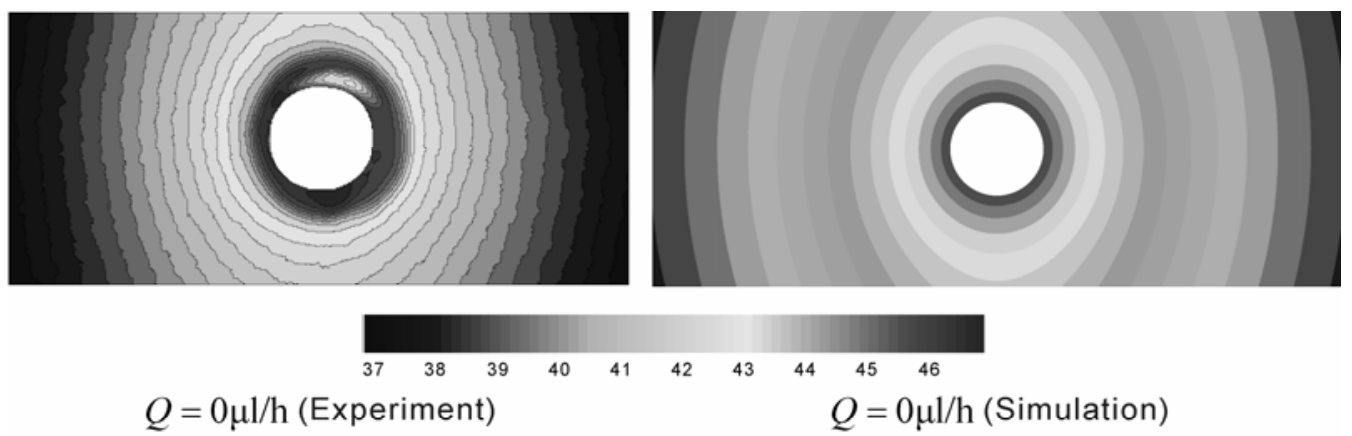

(a)

(d)
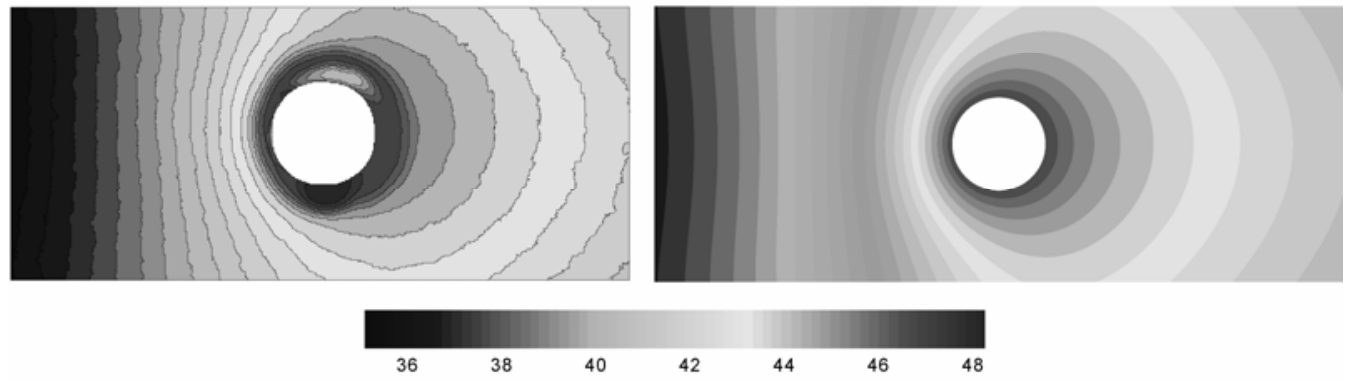

$Q=1000 \mu \mathrm{l} / \mathrm{h}$ (Experiment)

(b)
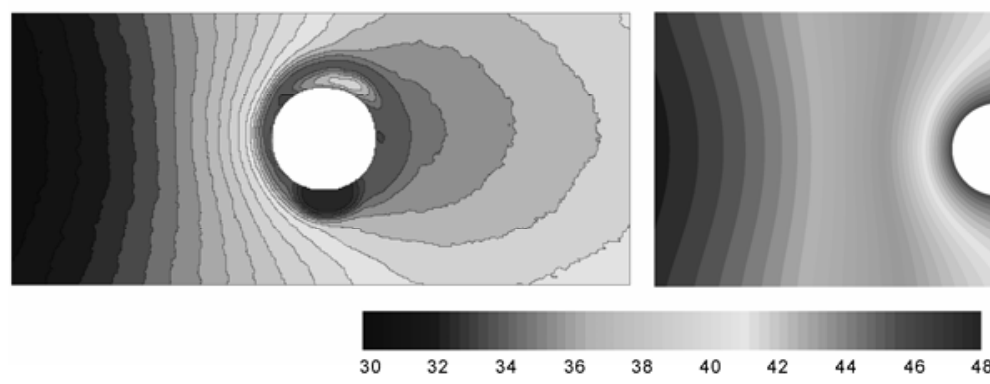

$$
Q=2000 \mu \mathrm{l} / \mathrm{h} \text { (Experiment) }
$$

(c)
$Q=1000 \mu \mathrm{l} / \mathrm{h}$ (Simulation)

(e)

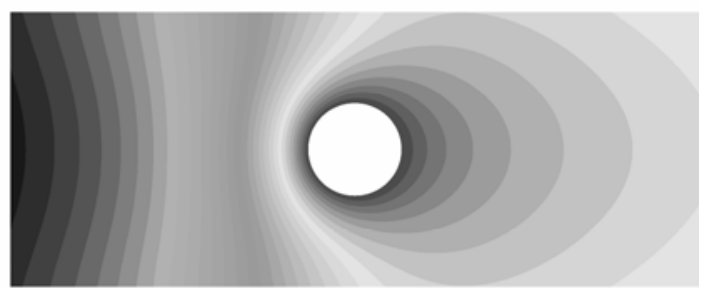

$Q=2000 \mu \mathrm{l} / \mathrm{h}$ (Simulation)

(f)

Figure 5. Experimental and numerical temperature distribution for different flowrates. (a)

$Q=0 \mu \mathrm{l} / \mathrm{h}$ (Experiment), (b) $Q=1000 \mu \mathrm{l} / \mathrm{h}$ (Experiment), (c) $Q=2000 \mu \mathrm{l} / \mathrm{h}$ (Experiment), (d) $Q=0 \mu \mathrm{l} / \mathrm{h}$ (Simulation), (e) $Q=1000 \mu \mathrm{l} / \mathrm{h}$ (Simulation), (f) $Q=2000 \mu \mathrm{l} / \mathrm{h}$ (Simulation) 


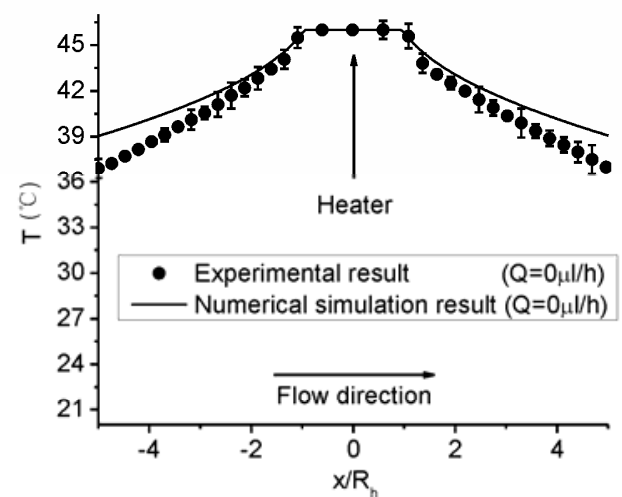

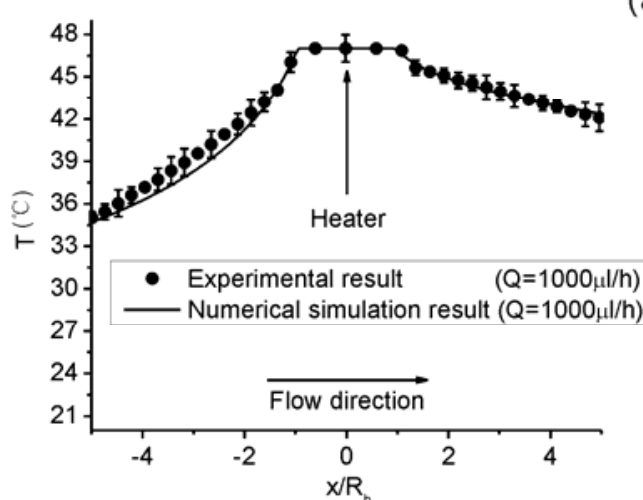

(b) (a)

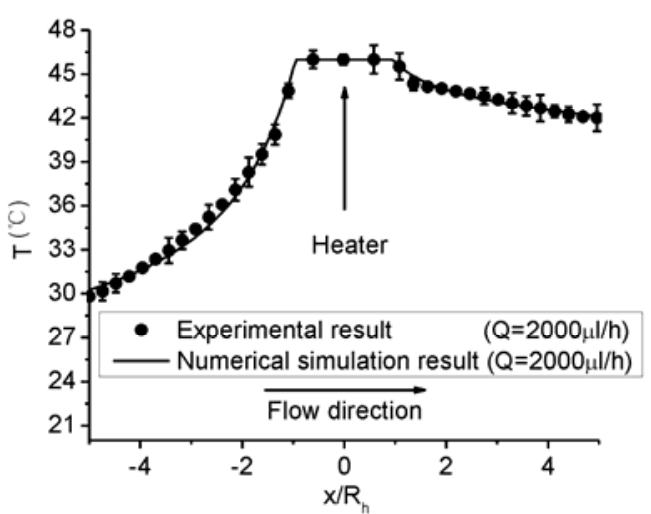

(c)

Figure 6. Comparison of the experimental and numerical simulation results along the central axis of the microchannel at different flowrates (a) $Q=0 \mu \mathrm{l} / \mathrm{h}$, (b) $Q=1000 \mu \mathrm{l} / \mathrm{h}$, (c)

$$
Q=2000 \mu \mathrm{l} / \mathrm{h}
$$




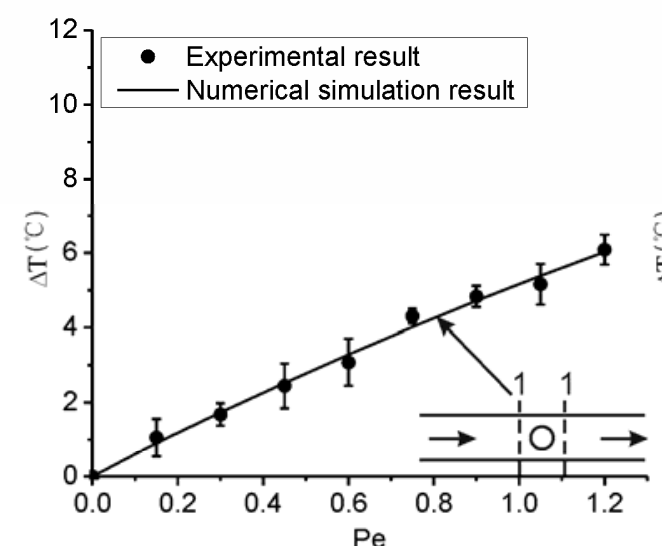

(a)

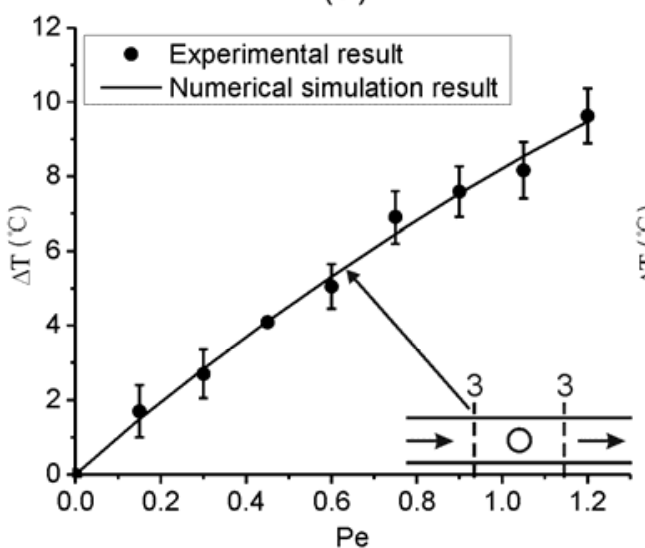

(c)

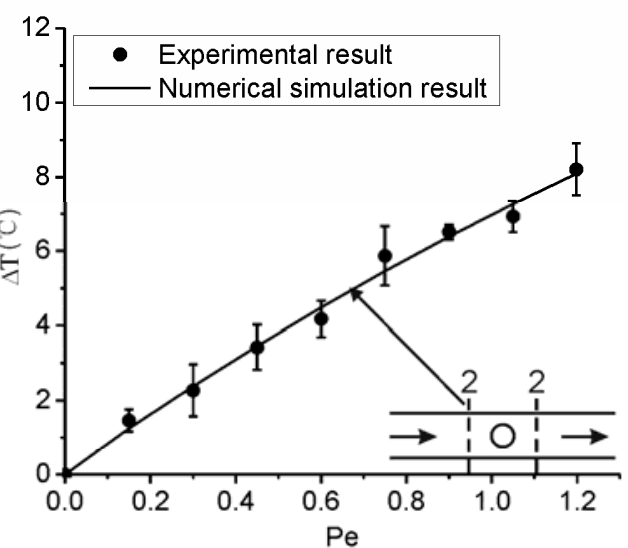

(b)

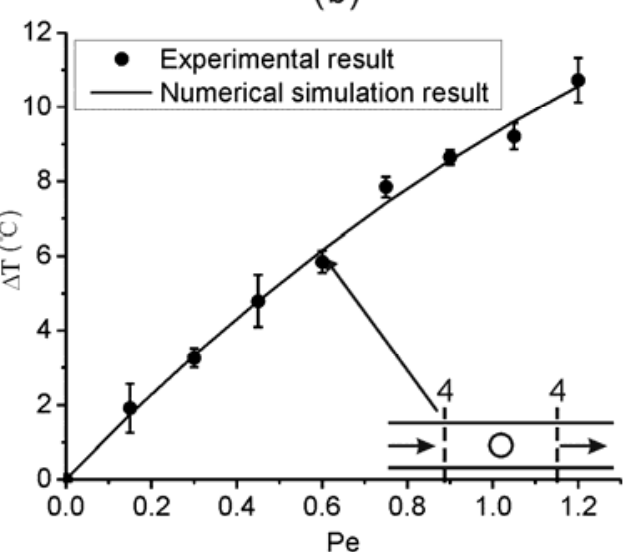

(d)

Figure 7. Comparison of the experimental and numerical simulation results of temperature

difference between two positions upstream and downstream (a) 1-1 section, (b) 2-2 section, (c) 3-3

section, (d) 4-4 section 